\title{
A cognitive and metacognitive approach for exploring link between urban transport environment and driver behaviour: case of roundabout crossed by tramway line
}

\author{
Fatiha Moutchou ${ }^{1}$, and Abdelghani Cherkaoui ${ }^{1}$ \\ ${ }^{1}$ Research team "Engineering, management and optimizationof Systems"(EMOSYS), Industrial Engineering Department, Mohammadia \\ School of Engineering, Rabat, Morocco, BP 765 Rabat agdal, Code postal 10100, Morocco
}

\begin{abstract}
The proliferation of roundabouts and tramway lines are among the most spectacular transformations of Moroccan roads and landscapes in recent years. These changes raise serious concerns about the effects of these new urban and road developments on traffic, on drivers' behaviours, and thus on road safety. These issues bring up the question concerning the adequacy bet ween the choices of road designers and the expectations of road users. Indeed, sustainable urban transport and well functioning road cannot be de signed without a holistic understanding of the way drivers act in changing environment through which they move in time and space. Consequently, there is a strong need to further knowledge about practices and behaviours of road users. The present research investigates the cognitive and metacognitive strategies use by drivers in a complex technical system such as roundabo ut crossed by tramway line. In this article we propose an empirical model to explore the effect of urban transport environment on drivers' behaviours. The paper presents an exploratory study based on a data triangulation app roach including database analysis, interview, observation, and think aloud protocol. The paper presents the main findings of the study and discusses their implications for road safety.
\end{abstract}

\section{Introduction}

The evolution of contemporary cities was characterized by changes in lifestyles and mobility practices. What is changing according to Nuvaloti [1], are the functions of the cities. Cities have become places where people go to work, consume, visit but less so to live.

In Morocco, due to the growth in population, the rapid urbanization, and the increase in daily mobility in the last decades, transport has become a highly strategic issue for urban areas. Indeed, if transport has shaped cities and guided their growth and their evolution, it constitutes today one of their major problems.

According to the World Bank assessment report on urban transport sector in Morocco, published in 2009 [2], «Public transport systems perform poorly and are not responsive to the changing demands of the population. The road infrastructure and the management of traffic are also lagging behind, and congestion is increasing fast $»$. So, solving problems of congestion or time lost in public transport call for a rethinking of mobility, particularly by redesigning road and expanding alternative transportation.

In Morocco, there has been much interest in tackling congestion and pollution in urban areas through the promotion of roundabouts and tramway lines. This choice comes often from the facility of turning back, the fluidity and accessibility that they bring. Indeed tram roundabouts are seen to be an effective tool in managing traffic flow in a safe and aes thetic manner.

But if these new infrastructure projects begin to develop today, their implementation in urban areas has lead to changes both in terms of urban shape, as on overall functioning of the city.

The roundabout crossed by a tramway is the intersection of the road and a tramway line. This refers to the definitions of 'the road', 'the railway', and their diversities. Tram-car interaction within the road network takes place in the context of existing road infrastructure, which has limited resource. But beyond this competition for space [3], the roundabout crossed by the tramway line is finally the interaction between two transport systems with contexts, constraints, and traffic rules specific to each of them.

Overall, it has to be stressed that is not only the built space, but also the lived and travelled space that must be taken into account to overcome major challenges that transportation and road safety present in urban area, and to make the system user friendly and well adapted to the qualifications and limitations of road users.

Research on road safety has long been focused on corrective approach after the occurrence of the accident. This approach has reached its limits. The current reflections are mostly focusing on issues relating to cohabitation and behaviours of urban road users. 
'Traffic calming' techniques implemented in Europe, in the late 1970's [4], and the "vision zero approach" developed in Sweden [5], are a good illustration of measures which aim to resolve traffic and safety problems with preserving and enhancing neighbourhood liveability. These approaches, focusing on the relationship between space and users practices, also open up interesting perspectives on the responsibility for safety which must be shared between those who design, and those who use the road transport system.

All these foreign experiences are of great interest, not to copy ready-made solutions, but to draw lessons from other countries that are very useful for the formulation of strategies and models adapted to the Moroccan context.

In this perspective, the question of the relationship between driver and his environment serves as a guide to our reflection. This study is concerned with aspects of urban environment that influence driver's behaviours. The focus is on the cognitive and metacognitive strategies of drivers when crossing a roundabout with tramway line.

In this article, we first introduce models of driving behaviour used in our analysis. Then central concepts and issues related to cognition, and metacognition are highlighted. With this as a background, we propose a model to study the interaction tram-car driver. We present in this paper our experimental study to collect data on driver behaviour when crossing roundabout with tram and we conclude with the main findings of our research and their implications for road safety.

\section{THEORETICAL FRAMEWORKS}

\section{1 urban environment complexity and drivers 'cognitive resources}

It is opportune to start this introductory literature review by recalling that driver in urban environnement faces an overflow of information coming from (others users, neighbourhood, infrastructures design, roads signs...). Thus, it comes back to driver ability to find the needed information from the plethora of information available.

As Healey [6] states, the challenge in urban regions is that an urban region or a city is not a "thing", but an imagined phenomenon, a conception of a very complex set of overlapping and intersecting relations, understood in different ways by different people. City changes all the time and so do the mental representation of the city. This approach gained insight from the work of Kevin Lynch [7], a planner who argued that each individual holds a unique "image" of his or her city, a visual representation that guides through daily life and maps out meaning.

Because the driver reactions cannot be changed, great attention is paid nowadays to the meaning of the space the driver is moving in. This area of research and action is known as "positive guidance"[8], "self explaining road" [9], and "road readability" [10] all these terms have been applied to those road environment which are in line with the expectations of the road users, eliciting safe behaviour simply by design. A malfunctioning urban environment is therefore identified by the fact that drivers do not behave as intended. The need to understand more about driver behaviour in relation to urban environment has guided our research to an approach that takes into account the Moroccan road context and the driver behaviours in this context.

\subsection{Role of cognition approach to identify drivers needs}

There is an expanding research literature examining the effect of urban environment on drivers' behaviours [1115]. The literature shows that in order to design a safe and well functioning road and urban development projects, we need to have a good understanding of how we operate as roads users. For this purpose, the cognitive approach is especially suitable for analysing human functions such as perception, mental representation, and decision making.

This study is based on two theoretical models that originate from cognitive psychology and are frequently mentioned in the literature on road user behaviour, namely Endsley's model of situation awareness, and the hierarchical structure of the driving task as described by Michon.

The concept of situation awareness proposed by Endsley [16] refers specifically to "the perception of elements in the environment within a volume of time and space, the comprehension of their meaning and projection of their status in the near future". Applying Endsley's model to the driving environment, the driver firstly perceives road situations. The driver then, has to understand what he has perceived by forming "a holistic picture" of the elements in the environment and their complex relationships. Finally, the driver projects his understanding into the near future, in order to take the most appropriate action. [17].

The concept of situation awareness focuses on the mental picture of the situation that people find themselves in, and how this picture can be distorted or improved by internal and external factors. These characteristics make it a suitable model for studying factors involved in unsafe or undesirable situations.

Also to better understand drivers' behaviours, the model of Michon [18] is a good starting point. Michon distinguishes three subtasks in driving with different temporal and cognitive requirements. The strategical level, related to planning trip (e.g., vehicle to use, route to take). The tactical level, that requires taking decisions about driving speed (e.g., passing a lead vehicle), and how to handle specific traffic situations such as crossing a crossroad. At the operational level, drivers exercise maneuvers allowing vehicle control (i.e., braking and steering).

Strategical level and tactical level have been found to be more cognitively demanding than operational level [19]. Indeed, at the strategic level, decisions are not really restricted by time, while at the tactical and even more so at the operational level, decisions are to be taken immediately. In this study we focus on the tactical level.

\subsection{Metacognitive strategies in a driving context}


The term "metacognition" is used to denote higher-order cognition about cognition, or "thinking about one's thinking" [20]. It refers to "the ability to understand and monitor one's own thoughts and the assumptions and implications of one's activities" [20,21]. The difference between metacognition and cognition is the conscious, or controlled, aspect of processes. Whereas, cognitive skills are needed to perform a task, metacognitive skills are necessary to understand how it was performed. The assumption underlying this distinction is that metacognition helps to improve performance [22, 23]. The person's awareness of his strengths and limitations allows an optimal selection of the strategies to perform a task, as well as an assessment of the gap between his performance and his objectives in order to correctly manage risk resulting from task complexity. Thus metacognition could provide us cues to examine what drivers are thinking and how they are acting. Research on metacognition has largely focused on topics of learning and teaching. However, little research has attempted to extend metacognition to driving context.

In this study, we borrow from the work of Reulier [23], her description of the activities carried out by a reader to control his understanding, to illustrate our analys is of drivers' metacognitive difficulties.

The table 1 below presents our taxonomy of metacognitive difficulties in driving context.

Table 1. Metacognitive difficulties in a driving context

\begin{tabular}{|c|c|}
\hline $\begin{array}{c}\text { Metacognitive knowledge } \\
\text { and strategies }\end{array}$ & Metacognitive difficulties \\
\hline Knowledge about person/self & $\begin{array}{ll}\checkmark & \text { Poor perception of one's } \\
& \text { own driving skills } \\
\checkmark & \text { Excessive self-confidence } \\
\checkmark & \text { Lack of self-confidence }\end{array}$ \\
\hline Knowledge about task & $\left\{\begin{array}{l}\checkmark \text { Lack of knowledge about } \\
\text { task features }\end{array}\right.$ \\
\hline Knowledge about strategies & $\mid \begin{array}{ll}\checkmark & \text { Lack of flexibility in the } \\
& \text { use of strategies } \\
\checkmark & \text { Lack of knowledge about } \\
\text { strategies }\end{array}$ \\
\hline $\begin{array}{l}\text { Metacognitive skills for } \\
\text { planning }\end{array}$ & $\begin{array}{ll}\checkmark & \text { Difficulties in the route } \\
\text { planning, choosing vehicle } \\
\text { or remembering itinerary }\end{array}$ \\
\hline $\begin{array}{l}\text { Metacognitive skills for } \\
\text { controlling and monitoring }\end{array}$ & $\mid \begin{array}{ll}\checkmark & \text { Lack of sensitivity to } \\
\text { inconsistencies in road } \\
\text { environment information }\end{array}$ \\
\hline
\end{tabular}

\begin{tabular}{|l|l|}
\hline $\begin{array}{l}\text { Metacognitive skills for } \\
\text { regulating }\end{array}$ & $\checkmark$ Failure of self-regulation \\
\hline
\end{tabular}

\section{Proposal of a model to assess the interaction "tram-car driver"}

Figure 1 contains the model we came up with to assess the interaction car-tram in roundabout. The articulation of models presented before seemed to us particularly interesting to fully understand human behaviour in typical driving situations such as roundabout crossed by tramway line. We also combine notions of spatial cognition to our analysis to suggest how cognitive mapping might be employed to help us better understand and predict driver behaviour.

The figure 1 below, represents a system composed of three spheres related to each other which are influenced by variables to maintain the equilibrium of the system. The successful adjustment or functional failures are regarded as the result of drivers' actions.

The three spheres can be described as follows:

Environment: the Environment to apprehend is considered as a system with its components and which is influenced by variables derived from Cauvin's work [24], such as places called landmarks or nodes (visibility, legibility, size, neighbourhood,) and links. (linearity, number of lanes, connections).

Driver: the driver is approached through the concept of "situation awareness" of Endsley [16]. The cognitive processes can be seen as a continuous cycle of perception, comprehension, projection and decision. In this study these processes are supposed to be influenced by the personal characteristics of the subject such as (metacognition, personality, expectation and motivation), and its status such as (age, gender and experience).

Action: the driver behaviour is studied through the actions (i.e. the driving task), divided by drawing inspiration from the model of Michon, in three level (strategic, tactic and operational). The drivers' actions are influenced by constraints related to activity goals (work, leisure...) as well as links with the space (traffic flow, mode of locomotion).

Thus, this framework allows generating and testing hypotheses about the specific causes of safe and unsafe driving behaviour. 


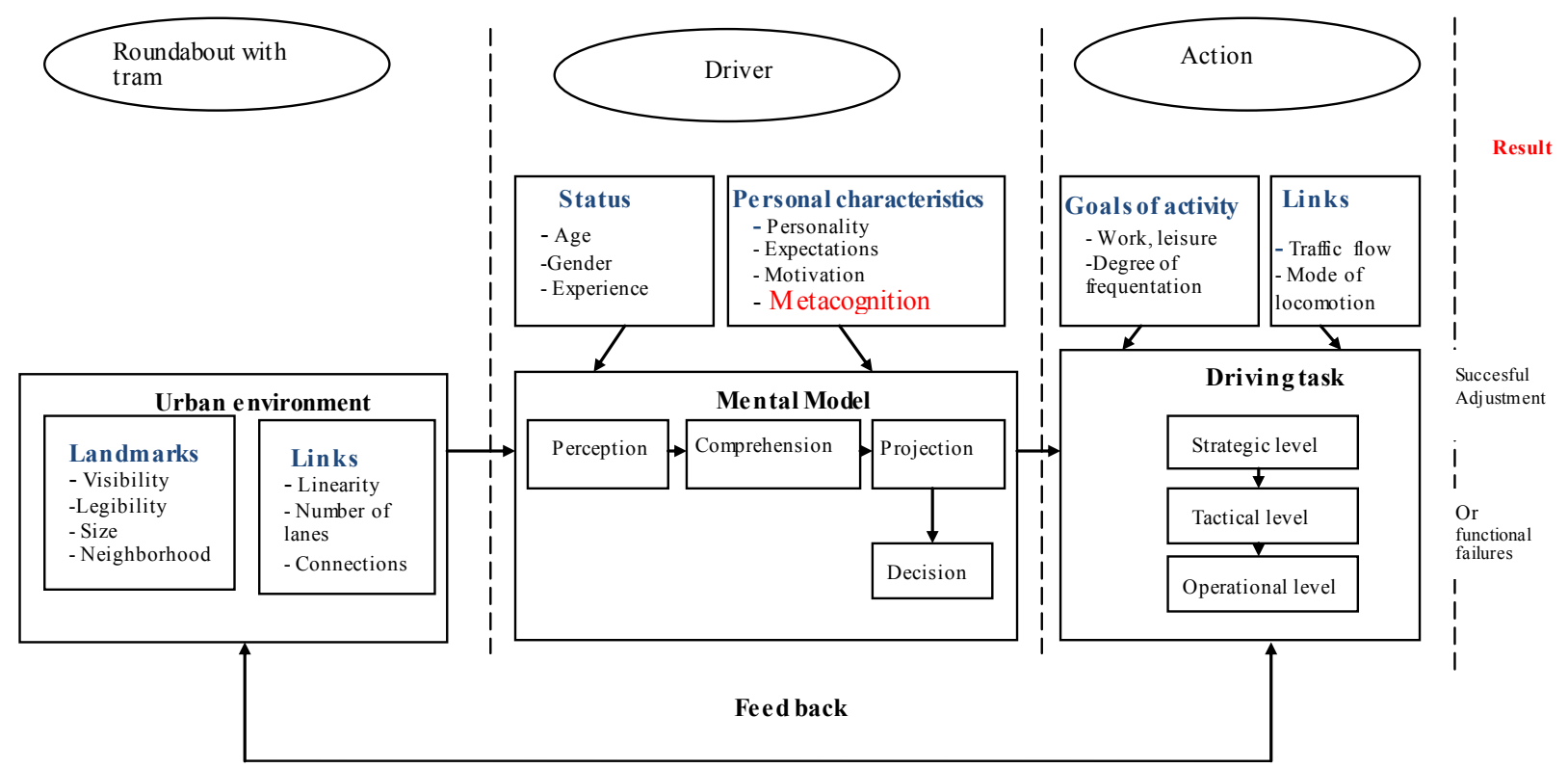

Figure 1. Framework for the tram-car driver interaction assessment.

\section{Research Method}

To validate our hypotheses, a particular attention is paid to the collection of data. For this purpose, our methodological proposal is based on the combination of quantitative methods (statistical analysis) and qualitative methods (related to drivers' expectations and their driving experiences). This requires implementation of several experimental protocols including accident database analysis, interview, observation, and think aloud protocol. The main advantage of this approach is that each event could be verified from different avenues.

\subsection{Tram accident analysis}

The Rabat Salé Tramway company (STRS) has collected data on accidents, between trams and others roads users, occurring in Rabat sale Tram network. Our analysis of the STRS accident database was carried out in order to have a global view on tram accidents, when, with whom and in which circumstances the accident happened.

The data used in our research covers the years 2011 to 2014. These four years, were chosen in order to have sufficient data to draw conclusions about a possible trend of evolution.

\subsection{Roundabout visits}

The choice of the roundabout studied named "IBN ROCHD Roundabout" was based on the number of accidents that happened there. Indeed there were 54 tram accidents between 2011 and 2014 at tram roundabouts. 17 of which were in "IBN ROCHD Roundabout".

Several visits, in vehicles and on foot, were carried out at the roundabout studied. This involved spending periods of time at the roundabout, observing users behaviours.

The observation was based on an analysis grid developed to carry out the assessment of factors that can affect the roundabout safety. The items included in this grid are elements of roundabout environment and users' behaviours which are inspired from several references studied [24] and detailed in our previous articles [3],[25].

\subsection{Interviews and think aloud protocol}

The experimentation was set up in two steps consisting of a verbal or think-aloud protocol and a retrospective interview.

- Verbal protocol: Twenty participants (eleven males and nine females) were part of the study. Participants were asked to carry out a think- aloud protocol by making a continuous dialogue of their internal thoughts related to their drive, and their progression through the roundabout studied. This involved drivers verbally expressing how they felt and making comments about their behaviours, especially as regards to the environmental conditions which in fluenced them and came into play in the various situations they encountered. An assessment of driver behaviours (speed, overtaking and driver's bodily expressions) was recorded on a grid developed beforehand.

- Retrospective interview: following the drive a retrospective interview was conducted. The participants were asked about their general impression and about particular points of the itinerary (signalling, visibility ....). 
In order to deepen our analysis, verbalization and interviews of vehicle drivers were completed by the questionnaire of the tramway drivers, and interview with tram operators, to investigate users' behaviour from tram drivers' viewpoint.

Two journeys within a tram driver's cab were also performed. Tram Drivers were asked to think aloud during the drive, and verbal assessments of the driving situations were recorded.

\subsection{Waiting time measures}

Perceived waiting time at tram roundabouts differs from the real waiting time. For the purposes of this study, we measured the real value of the cycle of traffic lights at the roundabout studied to gain more knowledge concerning the role of tram in influencing driver's perception of waiting time in roundabouts with trams.

\section{Findings}

\subsection{Design of the roundabout studied and visibility issues}

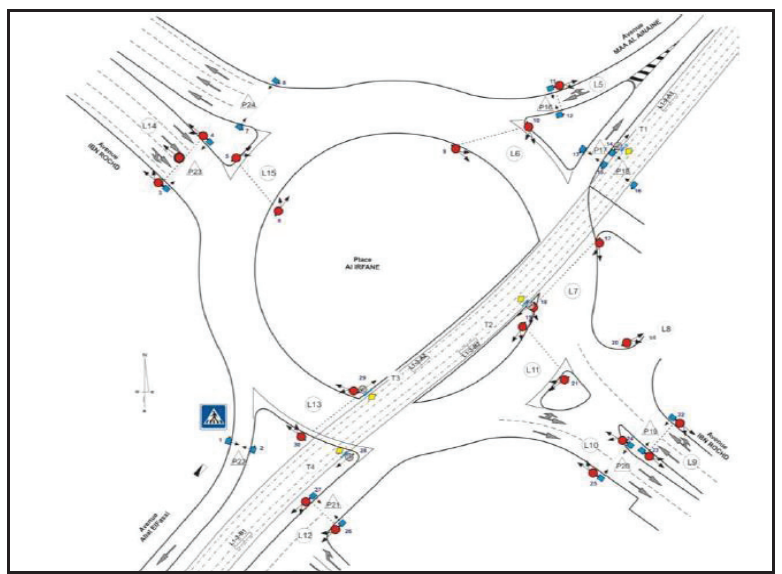

Figure 2. The lay out of the signalized roundabout under study "IBN ROCHD Roundabout" is a tram roundabout with four arms. The configuration is following: two lanes going into intersection and one separate lane for vehicles to turn right. Diagram of intersection geometry is in figure2.

Concerning the visibility of the roundabout studied, our analysis reveals the following shortcomings:

- At some locations there is considerable visual clutter from surrounding infrastructure, which can detract from the primary safety messages "give way to tramway" and "beware of tram";

-The curvature of the intersection creates a difficult angle from which to observe on-coming trams.

\subsection{The results of the analysis of STRS accident Database}

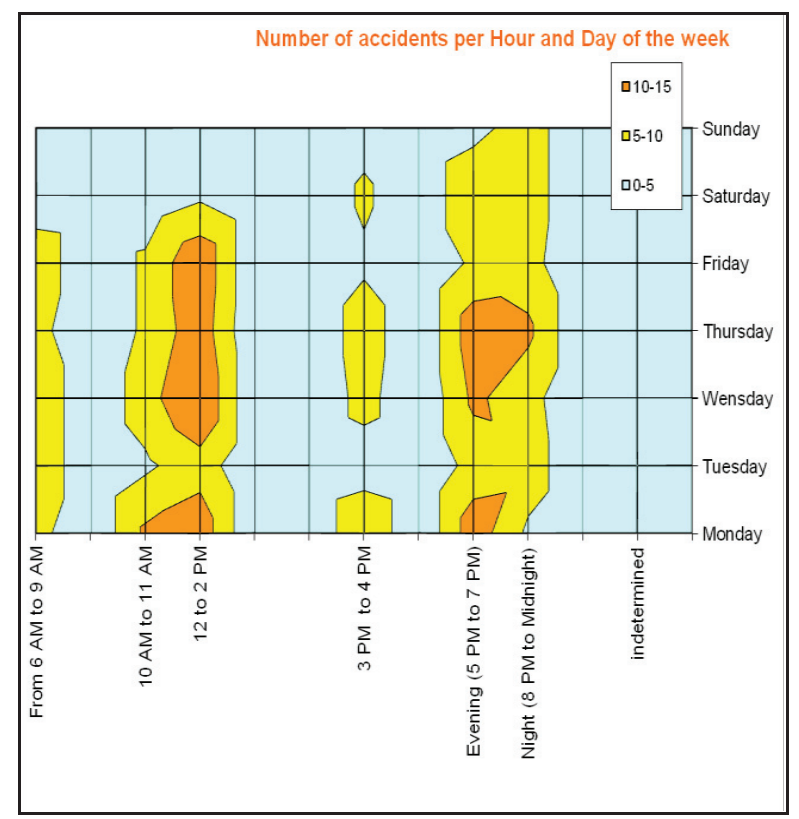

Figure 3. Number of accidents per hour and day of the week This graph shows that the highest number of accidents occurs during peak hours of lunch break and at the evening from Monday to Friday with the greatest proportion of crashes on Wednesday and Friday for the time slot between 12 p.m to 2 p.m.

This confirms the assumptions of our model and can be explained by the motivations of the drivers (the need to arrive at destination at time), and high workload. These findings are also to be expected due to traffic flow:

- Home-Based work trips that are more concentrated during these time slots;

- Most of private schools in Morocco close on Wednesday afternoons, and several parents must return to the school to pick up their children;

- Friday experiences also a very significant traffic flow for the time of the prayer from 12 p.m to 2 p.m.

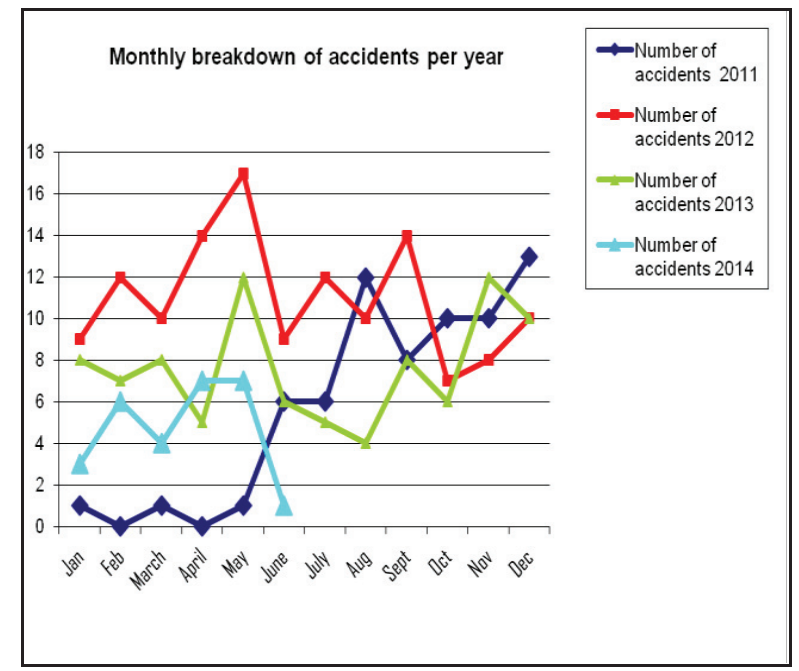

Figure 4. The monthly distribution of accidents 
The larger proportion of tram accidents occurs during the winter months of September and Dece mber as shown in figure 4. This is most likely due to problems of reduced visibility due to fog and rain. Concerning the high rate of accidents in May, it may be linked to the organization of an international festival during this time of year in the cities of Rabat and Sale, Morocco. This festival is experiencing a strong affluence of non-resident visitors. This finding is consistent with the assumptions of our model and is assumed to stem from the lack of familiarity of non resident drivers with tram roundabouts. So, the roundabout with tram line should be designed to meet drivers' expectations so that drivers know where they are (landmarks), and how to navigate in it (self explaining urban development).

\subsection{Metacognitive strategies of tram's drivers}

Most Tramway drivers questioned seem to perceive accidents and incidents as the results of factors over which they have no control. Their awareness of risks helps them to be more careful and to question some aspects of their know-how in order to better regulate their activities. The following are two examples of what some participants stated:

"After this accident, $i$ started to make better use of the rear-view mirrors and cameras".

"As you see, the driver hesitates to cross. I start by warning of the tram's approach using the warning horn, but at the same time, $i$ slow down a bit in case he would go through the red light".

The qualitative data analysis suggests that tram drivers are likely to encounter unsafe situations and need to anticipate other road users 'actions in advance to avoid conflicts. So, drivers have to use their metacognitive strategies to evaluate, to examine alternative sources or use context clues. Monitoring strategies are then, strongly associated with the ability to assess situations, to judge, or to make good decisions. The likelihood of occurrence of an accident is therefore greater when there is a lack of knowledge about strategies or lack of self-regulation.

\subsection{The drivers' ability to understand road signs}

The roads signs are very visible in the roundabout studied. Despite this, the results of our study show that they do not attract driver's attention because of the width of the road and the multiple solicitations of attention. The question of perception of road signs raises also the question of understanding of their meaning. If drivers do not perceive the tram signs, it is probably because they do not understand them. Indeed when roads signs don't fulfil an information need of the driver, he has no need to read them and therefore often ignore them. Indeed, this has been pointed out by one of the participants:

"I focus my attention on the traffic lights. I do not notice the tram road signs. To be honest, $i$ do not even look for them"

For many drivers there is no difference between all the tram signs. So, they cannot understand what is expected of them. It was also noted that tram warning signs were worded differently in Arabic and French. The sign "tram crossing the road ahead", is translated to "look out for trams". This discrepancy increases the ambiguity of the instruction.

These results confirm the assumptions of our model and can be explained by driver's situation awareness. Using their mental model, drivers try to perceive road signs that they believe are relevant while they just ignore those judged without useful information.

\subsection{Perceived waiting time}

According to the interviewees, the difficulties encountered in the roundabout studied are related to the cycle time of traffic signal.

If from our measures, the red light duration does not exceed 2 minutes even when the tramway is passing, drivers believe however that the average waiting time in the roundabout is about 15 minutes.

As Urry [26] points out, the classic view of travel time is basically built on the idea that "the time that is spent travelling is unproductive and wasted-dead time", and therefore a time to reduce.

The taxi drivers and the drivers with the experience of getting stuck seem to have the fear of congestion when the traffic is too crowded. Due to the number of stops experienced when queuing at the traffic light, drivers come to believe that a signal is dysfunctional, and they may try to run red light and to cross without the necessary caution. It was also stated that drivers alter their route choice to avoid getting stuck in the roundabout studied.

\subsection{Effects of roundabout environment on driver behaviour}

Many unfamiliar drivers with the area may not be expecting trams; the roundabout should provide information about trams using signage and surface delineation. From our analysis there is a number of design features at the roundabout studied that can play a role in unwanted events and contribute to unsafe conditions:

- stop line is not demarcated, either with a white line or appropriate pavement marking, to attract the driver's attention and to help drivers to identify safe stopping location;

- Parked cars before and after the tramway line may result in vehicle drivers slowing and being caught on the tracks while a tram approaches;

- The roundabout studied is a roundabout with traffic light; however signs giving priority to vehicles within the roundabout were maintained with traffic lights, which can lead to confusion and errors for unfamiliar drivers.

\section{Discussion}

The study has shown that our framework for the assessment of the interaction between tram and car driver has provided a good basis for the analysis of driver 
behaviour, and the causes of accidents in the roundabout studied. Indeed, results of our study have cast light on a number of problems associated with tram roundabouts and have explored some of the road design and human factors that contribute to the difficulties experienced by drivers at these locations. From our finding three key points must be highlighted:

- Cognitive and metacognitive processes

Metacognitive difficulties in crossing roundabouts with tram due to lack of strategies or lack of self regulation can place drivers at a greater risk of crash. Also no experienced or unfamiliar drivers with roundabout crossed by tram may be more prone to unsafe crossing.

It was acknowledged that most drivers will fail to read signs and consequently do not benefit from the information provided by these.

- Task challenges

The key challenges in crossing tram roundabouts is the goal of the activity (the need to arrive at work at time) which can place added stress and time pressure upon the driver and lead to risky driving in order to save time.

The traffic volume can add to the complexity of the task and may explain the distorted perceptions of waiting time in roundabouts with tram.

- Urban environmental factors

A number of characteristics of tram roundabouts are likely to exert some influence on driver behaviour such as: legibility and recognition of the traffic signs content.

Possible negative effects on driver safety from the roundabout design might occur from lack of pavement marking and the restriction of visibility

Since we cannot "redesign" the driver, one key finding of this study is that the urban environment must be designed to allow the driver to discern, to identify, and to choose easily in this environment the indices for the effective regulation of its activity

\section{Conclusion}

In a complex and multidimensional system such as tram roundabouts, forming an overall picture of the whole interactions and factors influencing drivers' behaviours is too challenging due to the need to take into account factors that are difficult to quantify.

The interest of this new approach is to contribute to a better understanding of the mechanis ms of perception and understanding of the road environment that lead to deviation or successfuladjustment.

Evidence has been found that road scene elements and traffic condition play a role in the difficulties that drivers encounter when crossing these intersections. This study has opened avenues of possibilities for further research in the area of urban design. Indeed these findings can help managers and road designers in their search for improvement of existing facilities, enhancement of those that seem relevant and finally designing of future infrastructure. Another future research opportunity is to investigate if these finding in roundabout can be generalized to the entire network by expanding our model in roundabout to other locations.

\section{References}

1. G. Nuvolati, Resident and Non Resident population: Types of conflicts. P.Pucci and M.Colleni (Eds.), Understanding mobilities for designing contemporary cities, Research for development. (2016)

2. World Bank. Morocco, Urban Transport Sector Development Policy Loan Project. Report Document AB4928,(2009).

3. F.Moutchou, A. Cherkaoui and E. El koursi, Human factors in the analysis of the Tram- car drivers at Intersections. Journal of civil engineering and architecture, Vol 8, Serial No 77, April (2014).

4. K. Kjemtrup and L. Herrstedt, Speed management and traffic calming in urban areas in Europe: a historical view. Accident Analysis \& Prevention Vo1.24, No. 1, pp 57-65,(1992).

5. J. Whitelegg and G. Haq, Vision zero: adapting a target of zero for road traffic fatalities and serious injuries. Stockholm environment institute, (2006).

6. P. Healey, Urban complexity and spatial strategies: towards a relational planning for our times. Taylor\$ Francis, (2007).

7. K. Lynch, The image of the city. Cambridge Massachussettes, MIT Press, (1960).

8. M. Millot, Analysis of the complex links between urban forms and road safety. Study report of CERTU (Centre for studies on road networks, transport, urban planning and public construction),(2004).

9. G.Weller and M.Dietz, Definition, comparison and evaluation of existing self-exp la ining road approaches in Europe. Deliverable N ${ }^{\circ}$ 1, SWOV, (2010).

10. F. Saad, In depth analysis of interactions between drivers and the road environment. 4th Workshop of International Cooperation on Theories and Concepts in Traffic Safety (ICTCT), Vienna, pp. 65-79,(1991).

11. P. Salmon, M. Regan and I. Johnston, 2006, Human Error and Road Transport: Phase Two-A Framework for an Error Tolerant Road Transport System. Monash University Accident Research Centre report, (2006).

12. G. Helmers, Explanatory model for road user behaviour: Implications for the design of road and traffic environment. Nordic human factors guideline, (2014)

13. M. Millot, Urban growth and road safety: the complex influence of urban forms. Ph.D. Thesis, National Advanced Civil Engineering School (ENPC), Department of Transport, Paris, December.9, (2003). (In French)

14. D. Fleury, Categorization of road scenes and safety from accident: analysis to typical representations. Intellectica $\mathrm{N}^{\circ} 15$, pp.97-123, (1992).(In French)

15. R. Davidse, Assisting the older driver, intersection design and in car devices to improve the safety of the older driver. Swov, Nederland, (2007).

16. M. Endsley and D. Kaber, Team situation awareness for process control safety and performance. Process Safety Progress 17 (1) 43-48, (1998).

17. B.Bailly, Drivers situation awareness: fundamentals aspects, methods, and tool development for drivers' training. $\mathrm{Ph}$.D. Thesis, Lyon 2 University, Specialty 
cognitive psychology, December.13, (2004). (In French)

18. J.A. Michon, A critical view of driver behavior models: what do we know, what should we do? In: $L$. Evans, R.C. Schwing (Eds.), Human Behavior and Traffic Safety, Plenum Press, New York, pp. 485-524, (1985).

19. G.M. Matthews, D.J. Bryant, R.Webb and J.L. Harbluk, 2001, Model for situation awareness and driving: Application to analysis and research for intelligent transportation systems. Transportation Research Record, (1779), (2001).

20. J.H. Flavell, Metacognition and cognitive monitoring - A new area of cognitive-developmental inquiry. American Psychologist, 34, 906- 91, (1979).

21. A.L. Brown, J.D. Bransford, R.A. Ferrara and J.C. Campione, Learning, remembering, and understanding. In J.H. Flavell, and E.M. Markman (Eds.), Handbook of child psychology: Vol. 3. Cognitive, (1983).
22. H. Patrick, Social self-regulation: Exploring the relations between children's social relationships, academic self-regulation, and school performance. Educational Psychologist, 32(4), 209-221, (1997).

23. J. Reulier, Verbal interactions between peers and development of metacognition in students with reading comprehension difficulties. Thesis, University of Quebec in Rimoski (2012). (In French)

24. An approach of intra-urban spatial cognition, http://cybergeo.revues.org (accessed Jan. 27, 1999).

25. F. Moutchou, A. Cherkaoui and E. El koursi, Factors influencing driver's behaviour at intersections crossed by tram. Proceeding of the 3rd International conference on road and rail infrastructure, CETRA 2014, Split, Croatia, pp 751-760, (2014).

26. J.Urry, Travelling times. European Journal of Communication Vol 21(3): 357-372, (2006). 